\title{
A collider for anyons
}

\section{Fractional statistics in anyon collisions}

Authors: H. Bartolomei, M. Kumar, R. Bisognin, A. Marguerite, J.-M. Berroir, E.

Bocquillon, B. Plaçais, A. Cavanna, Q. Dong, U. Gennser, Y. Jin, G. Fève

Science 368, 173-177 (2020)

\section{Recommended with a Commentary by Steven M. Girvin, Yale University}

Bartolomei et al. describe a two-particle interference experiment for fractional quantum Hall effect (FQHE) anyons that is sensitive to their fractional statistics and obtain results in excellent agreement with theoretical prediction [1]. A number of past experiments including single-particle interferometry and edge-state thermal transport have provided evidence for the expected fractional statistics of FQHE quasiparticles, but this novel two-particle interference experiment brings a new level of sophistication and direct evidence for the fractional statistics.

To understand fractional statistics, it is crucial to distinguish between the behavior of the quantum wave function under permutation of the particle labels versus the behavior under physical exchange of the particles. Identical particles naturally have identical masses, spins and interactions. Hence the Hamiltonian must be invariant under permutation of the labels on the particles. This symmetry tells us that the non-degenerate eigenstates of the Hamiltonian must be invariant (up to an overall phase) under permutation of the particle labels. Because permuting two labels and permuting the same two again leads to the original labeling, the only allowed phases under permutation are \pm 1 , corresponding to bosons or fermions. It is not always appreciated that this statement is true even for anyons! Anyons are (quasi)particles that are distinguished, not by their permutation statistics, but by the Berry phase acquired under actual physical exchange of two particles, i.e. their exchange statistics. Such Berry phases are well-defined if, for fixed positions of the quasiparticles, the system has an excitation gap. These phases form an abelian representation of the braid group which can be non-trivial in 2+1-dimensions. In more complex cases where there is a manifold of $N$ degenerate states associated with each position of the quasiparticles, the Berry phase is replaced by an $\mathrm{SU}(\mathrm{N})$ matrix acting within the degenerate subspace-a possibility that has led to interest in topological quantum computation.

Single-particle interferometers measure the interference between quantum amplitudes associated with a single particle traveling to the same final state via two different paths. An optical Mach-Zehnder interferometer (see Fig. 1) is an archetypical example. Twoparticle interference typically involves the fact that two final states that differ only by the permutation of the labels on the two particles are actually the same final state if the particles 
are indistinguishable. Since they are the same final state, the two quantum amplitudes for arriving at these states add coherently and therefore interfere. The Hanbury-Brown-Twiss (HBT) effect in quantum optics is the archetypcial 2-particle interference. In this effect two incoherent sources of light (e.g. the light from two different sides of a star) do not produce any detectable single-particle interference in detectors on the earth. Nevertheless there exists a novel interference effect that correlates the fluctuations in intensity observed in two detectors on earth. This is because, as illustrated in Fig. 1 there are two paths the two photons can take to create a coincidence in the two detectors. Variation of the interference with spatial separation of the detectors can be used to measure the angular diameter of stars even though their size is far below the diffraction limit of optical telescopes.

A beautiful analog of the HBT effect can be seen in cold atom experiments with two spatially separated Bose-Einstein condensates that have never been in contact and hence have no mutual phase coherence [2]. If the BECs are released from their traps, the atom clouds expand and eventually overlap. In the overlap region the observed atom density shows an oscillatory interference pattern with a random phase that varies from shot to shot of the experiment. The two-particle correlator $\left\langle n(\vec{r}) n\left(\vec{r}^{\prime}\right)\right.$ exhibits oscillations that are insensitive to this random phase, whereas the single-particle interference pattern in the density washes out upon ensemble averaging.

The Hong-Ou-Mandel effect, well known in quantum optics, is yet another two-particle interference effect that concerns what happens to two single photons incident on an optical beam splitter (see left panel of Fig. 2). If the two photons are indistinguishable (same frequency, same arrival time on each side of the beam splitter), the input state $\left.\left|\psi_{\text {in }}=\right| 11\right\rangle$ is transformed by a 50:50 beam splitter into the output state

$$
\left|\psi_{\text {out }}\right\rangle=\frac{1}{\sqrt{2}}[|02\rangle+|20\rangle]
$$

in which, remarkably, the two bosons always exit from the same port. To see why this is so, consider the fact that an ideal beam splitter performs a linear transformation on the mode operators given by

$$
\begin{aligned}
\left(\begin{array}{l}
a_{\text {out }} \\
b_{\text {out }}
\end{array}\right) & =S\left(\begin{array}{c}
a_{\text {in }} \\
b_{\text {in }}
\end{array}\right) \\
S & =\left(\begin{array}{cc}
t & r \\
-r & t
\end{array}\right) .
\end{aligned}
$$

In order to preserve the boson commutation relations of the output modes, the matrix $S$ must be unitary and, without loss of generality, we can take it to have the form shown above where $t, r$ are the (real, non-negative) transmission and reflection amplitudes respectively, with $|t|^{2}+|r|^{2}=1$, and the mode labels refer to the left panel of Fig. 2.

Since in the Hong-Ou-Mandel experiment the input modes each contain precisely one photon, we have: $\left|\psi_{\text {in }}\right\rangle=|11\rangle$. Then using the S matrix for a 50:50 beam splitter (which has $t=r=1 / \sqrt{2}$ ), it is straightforward to show that

$$
\begin{aligned}
\left\langle a_{\text {out }}^{\dagger} a_{\text {out }}\right\rangle & =\left\langle b_{\text {out }}^{\dagger} b_{\text {out }}\right\rangle=1 \\
\left\langle a_{\text {out }}^{\dagger} b_{\text {out }}^{\dagger} b_{\text {out }} a_{\text {out }}\right\rangle & =0,
\end{aligned}
$$



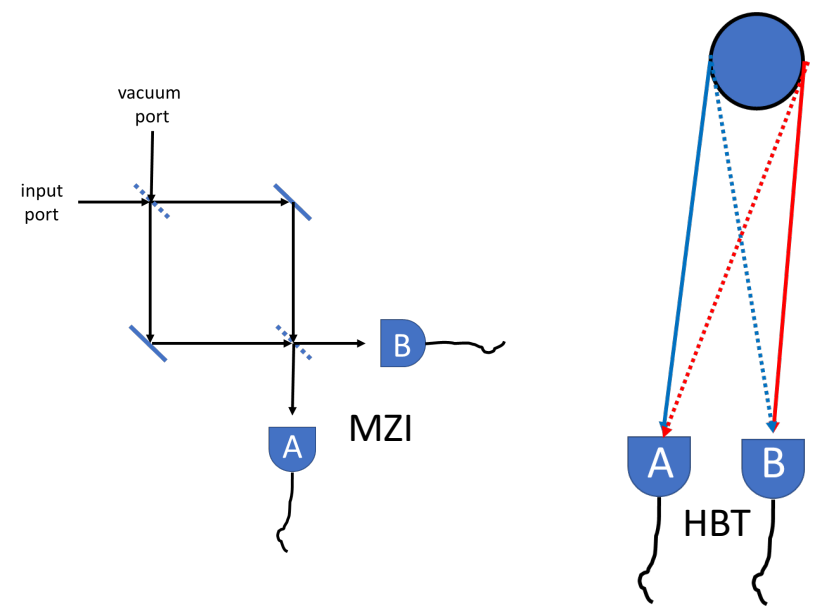

Figure 1: Left panel: A single particle entering the input port of the Mach-Zehnder interferometer can take two different paths to detector A. The interference depends on the path length difference of the two routes. Right panel: Hanbury-Brown-Twiss 'intensity interferometer' used for measuring the angular diameter of stars. Two photons, one from each side of the star, can take two different paths that will cause both detectors to fire. The two-particle interference does not require any coherence between the two photon sources.
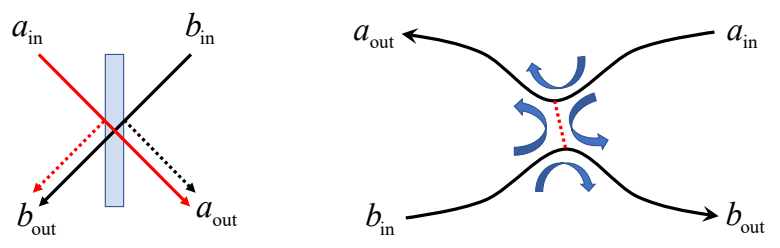

Figure 2: Left panel: Optical beam splitter that combines two input modes into two output modes. Right panel: quantum point contact 'beam splittter' that permits quasiparticle tunneling from one chiral edge mode to another in a fractional quantum Hall sample.

consistent with the fact that both photons always exit from the same port.

The experiment of Bartolomei et al. measures the cross correlations of the fluctuations in the number of particles exiting a quasiparticle beam splitter (quantum point contact) shown in the right panel of Fig. 2. Let us therefore compute the analogous quantity for our optical beam splitter by defining

$$
\begin{aligned}
\delta \hat{n}_{a} & =a_{\text {out }}^{\dagger} a_{\text {out }}-\left\langle a_{\text {out }}^{\dagger} a_{\text {out }}\right\rangle \\
\delta \hat{n}_{b} & =b_{\text {out }}^{\dagger} b_{\text {out }}-\left\langle b_{\text {out }}^{\dagger} b_{\text {out }}\right\rangle .
\end{aligned}
$$

Using Eqs. (3-4), we obtain the noise correlator

$$
\left\langle\delta \hat{n}_{a} \delta \hat{n}_{b}\right\rangle=-1 .
$$

Again this perfect anticorrelation is consistent with both particles always exiting the same port. 
If we repeat this calculation for the case of fermions we obtain

$$
\begin{aligned}
\left\langle a_{\text {out }}^{\dagger} a_{\text {out }}\right\rangle & =\left\langle b_{\text {out }}^{\dagger} b_{\text {out }}\right\rangle=1 \\
\left\langle a_{\text {out }}^{\dagger} b_{\text {out }}^{\dagger} b_{\text {out }} a_{\text {out }}\right\rangle & =1,
\end{aligned}
$$

meaning that two fermions can never exit the same port (because of Pauli exclusion) and so there must be one fermion in each output port every time. Thus

$$
\left\langle\delta \hat{n}_{a} \delta \hat{n}_{b}\right\rangle=0
$$

All of the results above for (free) bosons and fermions were derived considering only the permutation statistics of the particles. For the case of anyons we must recognize the role of the additional statistical phase $e^{i \phi}$ associated with physical exchange of the particles. For $\phi=0$, bosons continue to behave as bosons and fermions as fermions. For $\phi=\pi$ bosons act like fermions and vice versa. Thus as $\phi$ varies, we might naively expect $\left\langle\delta \hat{n}_{a} \delta \hat{n}_{b}\right\rangle=f$ with the parameter $f$ continuously interpolating between the limiting values 0 and -1 . The theoretical calculation in Ref. [1] addresses a number of non-trivial subtleties related to the fact that the 'anyon collider' of Bartolomei et al. employs gapless edge modes of FQHE states. These one-dimensional edge modes have long power-law tails in their temporal correlation functions. They are also driven out of equilibrium under the conditions of the experiment.

For the case of filling factor $1 / 3$ in the FQHE, quasiparticles have fractional charge $1 / 3$ and $\phi=\pi / 3$. Since this fractional charge constitutes less than half a fermion, we expect their behavior to be closer to that of bosons than fermions. In the limit in which auxiliary point contacts inject a low density of quasiparticle excitations into each of the two edge modes that meet at the primary point contact beam splitter (right panel of Fig. 2), Ref. [1] predicts that the cross-correlator of the currents in the two output ports will indeed be closer to the bosonic value than the fermionic. Bartolomei et al. find a generalized Fano factor (a suitably normalized measure of the cross-correlations) close to $P=-2$ in agreement with the theoretical prediction. The authors also carry out a number of control experiments and cross checks that confirm the result and are consistent with the detailed theory.

Readers interested in more details may wish to consult the commentary on Bartolomei et al. by Feldman [3]. New experimental results described in a recent preprint from the Manfra group [4], utilizing an electronic Fabry-Perot interferometer in which Coulomb charging effects are suppressed, will be reviewed in the accompanying commentary by Kivelson and Marcus [5].

\section{References}

[1] Bernd Rosenow, Ivan P. Levkivskyi and Bertrand I. Halperin, Phys. Rev. Lett. 116, 156802 (2016).

[2] M. R. Andrews, C. G. Townsend, H.-J. Miesner, D. S. Durfee, D. M. Kurn and W. Ketterle, Science 275, 637 (1997).

[3] D. Feldman, Science 368, 131 (2020). 
[4] J. Nakamura, S. Liang, G. C. Gardner and M. J. Manfra, https://arxiv.org/abs/2006.14115 (2020).

[5] Steven A. Kivelson and Charles M. Marcus, "At Last! Measurement of fractional statistics", Journal Club for Condensed Matter Physics, July 2020. DOI: 10.36471/JCCM_July_2020_02. 